\title{
The influence of diets on plasma homocysteine levels in felines
}

\author{
Mihaela COTUL ${ }^{1}$, Mihai CERNEA*2, Laura CĂTANA ${ }^{2}$ and Sanda ANDREI ${ }^{1}$ \\ ${ }^{1}$ Department of Preclinical Sciences, University of Agricultural Sciences and \\ Veterinary Medicine Cluj-Napoca, Romania. \\ ${ }^{2}$ Department of Clinic and Paraclinical Sciences, University of Agricultural Sciences and \\ Veterinary Medicine Cluj-Napoca, Romania. \\ * corresponding author: mihai.cernea@usamvcluj.ro
}

Bulletin UASVM Veterinary Medicine 77(2)/2020

Print ISSN 1843-5270; Electronic ISSN 1843-5378

doi:10.15835/buasvmcn-vm:2020.0023

\begin{abstract}
Epidemiological and clinical studies in humans established the relationship between homocysteine (Hcy) level in plasma and cardiovascular, kidney and liver diseases. In veterinary medicine, the results regarding Hcy are questionable due to conflicting and rare data. The relationship between the metabolism of Hcy and the nutritional principles of different diets can lead in the future to a real prevention of cardiovascular diseases by simply managing the food of predisposed and elderly animals. The present study aims at comparing two types of diets over a period of 9 months (commercial CD and homemade HMD) on the feline plasma Hcy. Analyzing comparatively the results obtained from the determination of serum Hcy values on the initial diets (t0) and after 9 months of diet ( $\mathrm{t} 1$ ), we can observe the decrease of these values both in group test I, which switched from a CD to a HMD, as well as in group test 2 , which maintained a CD but switched from a lower quality food to a higher quality one. This fact demonstrates not only the benefits of a home-made diet, but also the need to diversify and combine the commercial one to satisfy the nutritional requirements of the feline organism.
\end{abstract}

Keywords: homocysteine, alternative dietary habits, felines

\section{Introduction}

Homocysteine (Hcy) is an amino acid that contains a thiol group (-SH) and it is formed by intracellular demethylation of methionine. In the plasma, homocysteine is mainly bound to proteins (albumin), but free forms like oxidized ones and disulfide bonds are also present. Although, discovered in 1932 and extensively studied in human medicine as a plasma biomarker for multiple diseases (cardiovascular, cerebrovascular, renal, reproductive and genetic disorders), it is less required in veterinary plasma tests in Romania due to high costs (Vincent et al., 1952).

Not commonly found in mammalian diets, but it is an essential intermediate in the normal metabolism of methionine. Each of these compo- nents, methionine or homocysteine, is the precursor of the other. Similarly, the synthesis of one represents the detoxification metabolism of the other. This relationship is based on the ubiquitous presence of the methionine metabolic cycle in the organism (Finkelstein et al., 2000). Its two metabolic pathways influence its plasma levels. The first metabolic pathway is cysteine trans-sulfurization, in the presence of the enzyme cystathionine beta-synthetase (CBS), the enzyme that uses vitamin B6 as a cofactor. The second metabolic pathway is methionine remethylation via the enzymes methylene-tetrahydrofolate reductase (MTHFR) and methionine synthetase. The latter have as co-substrate folic acid (vitamin B9) and as co-enzyme, cobalamin (vitamin B12). 
Therefore, the plasma level of homocysteine is inversely proportional to the plasma level of folic acid, cobalamin and pyridoxal 5-phosphate (vitamin B6), being dependent on the exogenous intake of these nutrients (Selhub et al., 1999).

Hyperhomocysteinemia is defined as a pathological condition of excessive plasma homocysteine level (considered in humans to be at a plasma level above 12-15 $\mu \mathrm{mol} / \mathrm{L}$ ) and occurs in situations of blockage of one of the two metabolic pathways (Keung et al., 2015). Noting the increasing incidence of various pathologies in patients with hyperhomocysteinemia, in human medicine it has come to be considered as a relevant biomarker in the case of cardiovascular disease and myocardial infarction in geriatric patients. Furthermore, metabolic aberrations usually result in elevated plasma concentrations of Hcy and that is the reason hyperhomocysteinemia, along with folic acid deficiency, is considered a sensitive marker and also a risk factor in disorders such as cardiovascular, neurodegenerative and plasma clotting. As for veterinary medicine, studies and publications regarding Hcy as a marker for diseases and prognosis are scarce and the data found is conflicting. What we know for sure is that dogs suffering from heart and kidney pathologies have a statistically significant increase in this amino acid plasma levels compared to healthy dogs and that the use of it as a biomarker is highly requested (Gołnski et al., 2017). Hyperhomocysteinemia is usually the result of a sum of factors: genetic (polymorphism), physiological (age and sex), different drugs and dietary intakes (Verly-Jr et al., 2014). It has been shown that although hyperhomocysteinemia is a result of folate and/ or vitamin B12 deficiency, it also reflects an overall poor nutritional status correlated with low concentrations of vitamin $\mathrm{C}$ and carotenoids (Whalley et al., 2013; Celik et al., 2017).

The relationship between the metabolism of Hcy and the nutritional principles of different diets can lead in the future to a real prevention of cardiovascular diseases by simply managing the food of predisposed and elderly animals. Current studies show that there is a possibility that supplementation of the ration with folic acid and B-complex vitamins may regulate homocysteine metabolism without its accumulation at the systemic or cellular level, including the nervous system (Torin et al., 2007). However, high doses of the B vitamins, which are used to treat hyperhomocysteinemia, have possible side effects. Therefore, it is necessary to search for new alternative components to eliminate deleterious effects of hyperhomocysteinemia and to protect from hyperhomocysteinemia (Whalley et al., 2013; Celik et al., 2017).

Lately, pet owners have been looking for more natural or "home-made" diets detrimental to commercial or conventional ones, recommended by veterinarians. This tendency began at the same time with people's desire to move towards more natural and organic products in their own diet. We tried to understand both sides of the story, as owners and veterinarians and to offer ourselves science-based answers, hence the motivation to start this scientific study. Nowadays, veterinary medicine is shifting its attention towards prevetion more than ever and this motivates not only veterinary specialists but also owners to invest in a complete and high quality diet to prevent diseases. Why is Hcy the perfect biomarker for this study? The answer lies in the relationship between its metabolism and the effects of hyperhomocysteinemia in cats (Ozkan et al., 2017).

The present study aims at comparing two types of diets (commercial and homemade) on the plasma homocysteine level, diets maintained over a period of about 9 months.

\section{Materials and methods \\ Animal Selection}

The animals considered for this study were cats recruited via the students of the university. Information on the cats' environment and dietary history were obtained during a discussion with the owners, in which they were also asked about the presence of any sign of disease and ongoing drug treatments. The animals were clinically healthy with no gastrointestinal signs, polyuria/ polydipsia, neurological disorders etc. Each subject benefitted from a complete physical examination, plasma and urine testing.

The selected cats - in a total of five - were distributed into groups accordingly:

- Control group (C) with one subject (female, 4 years old) with outdoor access and a mixed diet;

- Test group 1 (T1) with two indoor subjects (females, 7 and 9 years old respectively) commercially-fed with a low quality diet. Both 
females had gingivitis at the start of the study and the 9 year old subject is a number 7 on the 9 -scale body score condition;

- Test group 2 (T2) with also two indoor subjects (females, 2 and 3 years old respectively) commercially-fed with a medium quality diet.

\section{Types of diet}

Each group was changed to a specific diet from the ones they were fed before, except control group that was maintained on a standard mixed diet. The composition of the diets selected for the study will be presented in the table 1 .

\section{Study Design}

Samples taken at the beginning of the study ( $\mathrm{t} 0$ ) and after 9 months of diet ( $\mathrm{t} 1$ ) were transported in ice to a laboratory, centrifuged, and homocysteine was determined from the serum by the enzymatic method.

\section{Plasma homocysteine determination}

Measurements of HCY were performed by ERI VET LAB Cluj-Napoca with an enzymatic kit (Diazyme). In brief, oxidized homocysteine (HCY) is reduced to a free form which reacts with S-adenosylmethionine (SAM) to form methionine and S-adenosylhomocysteine (SAH). $\mathrm{SAH}$ is hydrolyzed into adenosine. The generated adenosine is immediately dehydrogenated into inosine and ammonia. In the last step, glutamate dehydrogenase (GLDH) catalyzes the reaction of ammonia with 2-oxoglutarate and $\mathrm{NADH}$ to form $\mathrm{NAD}^{+}$.

Table 1. The composition of the diets for every group test selected

\begin{tabular}{|c|c|c|}
\hline Control group & Test group 1 & Test group 2 \\
\hline \multirow{4}{*}{ Standard Mixed Diet } & Home-made diet (HMD) & Comercial diet (CD) \\
\hline & $\begin{array}{l}\text { Composition: } \\
\text { - proteins from poultry } 61,8 \% \text {, } \\
\text { from fish } 44 \% \text { salmon and } \\
50 \% \text { tuna; - carbs } 11 \% ; \\
\text { - saturated fatty acids } 6 \% \text {; } \\
\text { - fibers } 6 \%\end{array}$ & $\begin{array}{l}\text { Composition: } \\
\text { - proteins } 27 \% ; \\
\text { - fats } 13 \% ; \\
\text { - fibers } 4 \% ; \\
\text { - Ca } 1,01 \% ; \text { Mg 0,07\%; } \\
\text { - biotin } 1.41 \mathrm{mg} ; \text { Fe } 216 \mathrm{mg}\end{array}$ \\
\hline & \multirow{2}{*}{$\begin{array}{l}\text { Added probiotics from home-made } \\
\text { yogurt (Lactobacillus acidophilus; } \\
\text { Streptococcus thermophilus) }\end{array}$} & $\begin{array}{l}\text { Mixed types (digestive, } \\
\text { sensible, hair\&coat, indoor, } \\
\text { sterilised and hairball) }\end{array}$ \\
\hline & & Higher quality and a higher price \\
\hline
\end{tabular}

\section{Results and discussions}

The results obtained in the determination of Hcy from the serum during the study are presented in table 2, along with the two reference values considered. The first reference values considered were determined in the study on levels of serum homocysteine, vitamin B12 and folic acid in healthy cats of the Van Turkish breed; study conducted by Özkan et al. (2016). This study, although grouping subjects by both sex and age categories, is limited by the fact that there are differences in the two breeds studied, Van Turkish vs. European breed from this study. Thus, values may differ depending on breeds. The second round of reference values considered are determined by Freeman et al. (2009), in the study on healthy cats, cats with cardiomyopathy or arterial thromboembolism. This study reveals comparatively different values of plasma homocysteine and B-complex vitamins. The study does not mention either the sex or the age of the animals included, the main criteria for choosing the subjects being the pathologies described above.

In the first determinations of homocysteine (t0), allanimalshadaslighthyperhomocysteinemia, compared with the reference values determined by age and sex in the study of Ozkan et al., 2017, on cats of the Van Turkish breed. The comparative analysis of their reference values with those of healthy cats from the study of Freeman et al., 2009, revealed that all cats maintain hyperhomocysteinemia at the upper limit of the maximum permitted levels 
of homocysteine and some cats have values much closer to those recorded at those at high risk of arterial thromboembolism.

For the age and sex reference values, subject 3 (T2) had the most worrying value of hyperhomocysteinemia, exceeding the maximum value by 5 nmoles/mL, and control subject and subject 2 (T1) were the most close to the maximum accepted values exceeding by only 0.2 and 0.4 nmoles/mL respectively. Taking into account the second study, Freeman et al. 2009, cited for the reference values, subject 2 (T1) recorded values below the maximum accepted level with a difference of 0.7 nmoles $/ \mathrm{mL}$, the control subject was at the upper limit, and subject 1 (T1) and subjects 3 and 4 (T1) exceeded the maximum value by $0.8 \mathrm{nmoles} / \mathrm{mL}, 0.7$ and $0.5 \mathrm{nmoles} / \mathrm{mL}$, respectively (Table 2 ).

The results of the study obtained after the establishment of the diets for 9 months recorded a slight decrease in all subjects: control 0.2 nmoles/ $\mathrm{mL}$, subject 1 (T1); 0.5 nmoles $/ \mathrm{mL}$, subject 2 (T1); 0.1 nmoles/mL, subject 3 (T2); 0.6 nmoles/ $\mathrm{mL}$ and subject 4 (T2) $0.3 \mathrm{nmoles} / \mathrm{mL}$. The most significant decreases are in subjects 1 (T1) and 3 (T2), subjects who recorded high values before their transition to the diets corresponding to the groups.

Comparing these results with the reference values of the first cited study, reveals a worrying increase over the maximum value of subject 3 (T2) by $4.4 \mathrm{nmoles} / \mathrm{mL}$ and a considerable improvement for control and subject 2 (T1). Considering the second cited study, the reference value for healthy cats places the control and subject 2 (T1) below the maximum values, while subject 1 (T1) and subject 3 and 4 (T2) again exceed the maximum value by $0.3 \mathrm{nmoles} / \mathrm{mL}$, respectively $0.1 \mathrm{nmoles}$ / $\mathrm{mL}$ and $0.2 \mathrm{nmoles} / \mathrm{mL}$. If this comparison takes place with the reference value for cats with arterial thromboembolism, subjects 1,3 and 4 exceed the maximum value before the diet, so that after 9 months they fall below the maximum value. None of the subjects exceeded the reference value for cats suffering from cardiomyopathy when determining both homocysteinemia at $\mathrm{t} 0$ and $\mathrm{t} 1$.

The calculation of the statistical index P (T1 p $=0.37 ; \mathrm{T} 2 \mathrm{p}=0.20$ ) reveals that it is insignificant due to the small number of subjects tested, being necessary for the future a larger study with a higher number of subjects of different ages, races or clinical conditions (example: pregnant females).

Because it is a new field, little explored and yet with a fairly impressive number of ongoing studies worldwide, we have chosen to study the relationship of homocysteine with different diets of cats, whether commercial or home cooked, and the comparison of the two in terms of nutrition. As mentioned before, homocysteine is a possible plasma marker for cardiovascular disease and myocardial infarction, especially in geriatric animals, too little researched in veterinary medicine. At present, it is considered that these diseases represent a third of the total deaths worldwide, and the prevalence is still increasing, being in the top 10 pathologies that affect cats.

Comparative analysis of homocysteinemia values at $\mathrm{t} 0$ and $\mathrm{t} 1$ for group test 1 , values that decreased by an average of 0.3 nmoles $/ \mathrm{mL}$, together with the clinical changes observed throughout the study showed the benefits of a home diet according to their own prescription. The relationship between the household diet and the weight regulation of the subjects requires

Table 2. Homocysteine values before ( $\mathrm{t} 0$ ) and after ( $\mathrm{t} 1$ ) 9 months of specific diet in tested subjects compared to reference values

\begin{tabular}{lcccc}
\hline Groups & $\begin{array}{c}\text { Hcy t0 } \\
\text { (nmoles/mL) }\end{array}$ & $\begin{array}{c}\text { Hcy t1 } \\
\text { (nmoles/mL) }\end{array}$ & $\begin{array}{c}\text { Reference 1 } \\
\text { (nmoles/mL) }\end{array}$ & $\begin{array}{c}\text { Reference 2 } \\
\text { (nmoles/mL) }\end{array}$ \\
\hline Control & 7.7 & 7.5 & $<7.5$ \\
\hline \multirow{2}{*}{ Test group 1 } & 8.4 & 7.9 & $<6.5$ & \\
\cline { 2 - 4 } Test group 2 & 6.9 & 6.8 & $<6.5$ & \\
& 8.3 & 7.6 & $<+/-4.1$ \\
\cline { 2 - 4 } & 8.1 & 7.8 & $<.3$ & \\
\hline
\end{tabular}


the thought of a future study for a possible diet suitable for sterilized / neutered cats with a tendency towards obesity. Whereas, the same analysis for group test II, with values decreased by an average of $0.45 \mathrm{nmoles} / \mathrm{mL}$, showed that a single type of commercial food does not bring the necessary nutritional intake to the feline body, requiring a food of superior quality and of several types (indoor, sterilized, hair \& coat, etc.). Comparing the values of both test groups with multiple reference values have shown the increased risk of subjects (especially subject 3 and 4 on the $\mathrm{CD}$ ) to cardiovascular pathologies, although clinically the subjects were declared healthy, approaching the maximum values for cats with arterial thromboembolism.

In addition to the serum determinations of homocysteine, for $\mathrm{T} 1$, certain clinical improvements of the two subjects were observed during the period of the home diet. Subject number 1 managed to balance its weight from $6.4 \mathrm{~kg}$ to $5 \mathrm{~kg}$, a change that may be the basis of a future study related to the household diet and its relationship with sterilized females prone to obesity. For both subjects, the number of meals decreased from four meals to two a day, demonstrating the possibility that the home diet would be more filling. Instead, there was an increase in water intake through the behavior of water-seeking cats; after using a bowl of water next to food, the tendency to hydrate them was observed after each meal. The temperament became lively (the two subjects played more often, walked, interacted with each other and with the owner) from a sedentary lifestyle in which the subjects slept most of the time. Another major and observable change was the lack of halitosis in both subjects and the increase in time between tartar removals. They were deducted about two months before the start of the study and a second is still not needed. Diminishing hair loss and regenerating the shine of the fur show that the nutritional intake from the HMD, according to our own recipe, can have a beneficial effect in regulating hair and hair ingestion by grooming; ingestion that predisposes to the formation of well-known "hairballs" responsible for regurgitation. The last observed clinical change is related to the previous one by the cessation of sporadic regurgitations after copious meals, regurgitations that improved only under drug treatment with Motilium. Stopping the drug treatment and solving the problem only with diet is a huge benefit for the subjects and the owner.

The published literature indicates that homocysteine is an independent risk factor for cardiovascular disease, modifiable through diet and exercise. However, it is now widely accepted that food sources, on their own, cannot consistently provide the levels of nutrients needed to support optimal homocysteine metabolism. In fact, emerging studies are uncovering new nutritional strategies for lowering high levels of homocysteine by offering new possibilities for preventing cardiovascular disease. Speculation about this special correlation continues to contribute to the perplexity of scientific society. Although most research suggests a relationship, there seems to be other evidence that still prevents it from being included as a biomarker. Every ten steps forward, we may face a step or two backwards, but this should further increase the enthusiasm for research in this area, which certainly requires more research until definitive evidence is available for remove any shadow of doubt about the correlation between homocysteine and cardiovascular disease. However, this review should provide some insight into the role of homocysteine in the development of cardiovascular disease, which summarizes both centrally and peripherally the effects of homocysteine and the relationship of the two to the type of diet imposed on animals.

\section{Conclusion}

Analyzing comparatively the results obtained from the determination of serum homocysteine values on the initial diets ( $\mathrm{t} 0$ ) and after 9 months of diet ( $\mathrm{t} 1$ ), we can observe the decrease of these values both in group test I, which switched from a CD to a HMD one, as well as in group test 2, which maintained a CD but switched from a lower quality food to a higher quality one. This fact demonstrates not only the benefits of a home-made diet, but also the need to diversify and combine the commercial one to satisfy the nutritional requirements of the feline organism.

Acknowledgement. This research project was supported by research grant no.37PFE/06.11.2018 


\section{References}

1. Celik N, Vurmaz A, Kahraman A (2017). Protective effect of quercetin on homocysteine-induced oxidative stress. Nutrition 33:291-296.

2. DuVigneaud V (1952). A trail of research in sulfur chemistry and metabolism, Cornell University Press, Ithaca, 21

3. Finkelstein JD, Martin JJ (2000). Related papers: molecules in focus. Homocysteine. International Journal of Biochemisrty \& Cell Biology, 32:385-389.

4. Freeman LM, McMichel MA, Selhub J, Rozanski EA, Brown DJ, Nadeau MR, Rush JE (2000). Plasma Homocysteine, B Vitamins and Aminoacid Concentrations in Cats with Cardiomyopathy and Arterial Thrombembolism. J Vet Intern Med, 14: 507-512.

5. Ganguly P, Alam FS (2015). Role of homocysteine in the development of cardiovascular disease. Nutr J, 14, 6 . https://doi.org/10.1186/1475-2891-14-6

6. Gołnski M, Lutnicki K, Krumrych W, Szczepanik M, Gołynska M, Wilkołek P, Adamek Ł, Sitkowski Ł, Kurek Ł (2017). Relationship between Total Homocysteine, Folic
Acid, and Thyroid Hormones in Hypothyroid Dogs. J Vet Intern Med, 31:1403-1405.

7. Keung W, Lai C, Kan MY (2015). Homocysteine-Induced Endothelial Dysfunction. Ann Nutr Metab, 67:1-12, https://doi.org/10.1159/000437098.

8. Özkan C, Kozat S, Kaya A, Akgul Y (2017). Serum homocysteine, vitamin B12 and folate levels in healthy Turkish Van Cats. Journal of Advanced Veterinary and Animal Research Turkey, 4(1):58-64.

9. Selhub J (1999). Homocysteine metabolism. Jean Mayer USDA Human Nutrition Research Center on Aging, Tufts University, Boston, Massachusetts, 19:217-240.

10. Verly-Jr E, Steluti J, Fisberg RM, Marchioni D (2014). A Quantile Regression Approach Can Reveal the Effect of Fruitand Vegetable Consumption on Plasma Homocysteine Levels. PLoS ONE 9(11): e111619. doi:10.1371/journal. pone. 011161.

11. Whalley LJ, Duthie SJ, Collins AR, Starr JM, Deary IJ, Lemmon H, Duthie AC, Murray AD, Staff RT (2013). Homocysteine, antioxidant micronutrients and late onset dementia. Eur J Nutr, 53:277-285. 